\title{
Efeitos do treinamento muscular respiratório em pacientes após acidente vascular cerebral: uma revisão sistemática
}

\author{
Effects of respiratory muscle training in \\ patients after cerebral vascular accident: a \\ systematic review
}

\author{
Fleury Ferreira Neto ${ }^{1}$ (i) \\ Jedson dos Santos Nascimento ${ }^{2}$ (1) \\ Ana Carla Cruz de Jesus ${ }^{3}$ (i) \\ Luiz Barauna 4 (1) \\ Nildo Manoel da Silva Ribeiro ${ }^{5}$
}

\begin{abstract}
1,3,4Centro Universitário Social da Bahia (Salvador). Bahia, Brasil.ffneto@faculdadesocial.edu.br, anacarla.cruz@yahoo.com.br, luiz.barauna@gmail.com ${ }^{2}$ Autor para correspondência. Centro Universitário Social da Bahia (Salvador). Bahia, Brasil. jedson.santos1990@gmail.com

5Universidade Federal da Bahia (Salvador). Bahia, Brasil. nildoribeiro67@gmail.com
\end{abstract}

RESUMO | INTRODUçÃO: O Acidente Vascular Cerebral (AVC) é o resultado da interrupção aguda do suprimento sanguíneo ao cérebro por meio de entupimento ou rompimento dos vasos podendo provocar lesões celulares e alterações nas funções neurológicas. Essas alterações são relevantes para a ineficiência dos mecanismos orofaríngeos da deglutição, respiração, fonação e reflexos protetores das vias aéreas inferiores culminando em alta incidência de pneumonia por aspiração. O Treinamento Muscular Respiratório tem como função restabelecer a função muscular que esteja comprometida, treinar tanta a força quanto à endurance muscular, melhorar a eficácia na desobstrução das vias aéreas, condicionar e adaptar ao exercício e prevenir a fadiga muscular respiratória. OBJETIVO: O objetivo desse estudo é sistematizar o efeito do treinamento muscular respiratório sobre os volumes e capacidades pulmonares em indivíduos que sofreram acidente vascular cerebral. MATERIAIS E MÉTODOS: Esta revisão sistemática foi realizada pela busca nas bases de dados BVS (Scielo, Lilacs, IBECS, Medline), PubMed e análise das referências. RESULTADOS: Um total de 4834 estudos foram rastreados e analisados, onde fizeram parte desta revisão 17 estudos publicados entre 2010 e 2019. CONCLUSÃO: Estudos comprovaram que o uso de TMR em pacientes pós-AVC gera efeitos positivos nos volumes e capacidades pulmonares, apesar de terem um tamanho amostral pequeno. A busca por criar novos estudos que possam auxiliar na prescrição e padronização dos treinamentos desses indivíduos em diversos ambientes deve ser um caminho a se pensar.

PALAVRAS-CHAVE: Exercício para os músculos respiratórios. Acidente vascular cerebral. Exercícios respiratórios.
ABSTRACT | INTRODUCTION: Stroke is the result of the interruption of blood supply to the brain and can cause cellular damage and changes in neurological functions. These changes are relevant to the inefficiency of the oropharyngeal mechanisms of swallowing, breathing, phonation and protective reflexes of the lower airways culminating in a high incidence of aspiration pneumonia. Respiratory Muscle Training has the function of restoring muscle function that is compromised, training both strength and muscle endurance, better effectiveness in clearing the airways, conditioning and adapting to exercise and preventing respiratory muscle fatigue. OBJECTIVE: The aim of this study is to verify the effect of respiratory muscle training on individuals who have suffered a stroke. MATERIALS AND METHODS: This systematic review was carried out by searching the VHL databases (Scielo, Lilacs, IBECS, Medline), PubMed and analyzing the references. RESULTS: A total of 4834 studies were screened and analyzed, where 17 studies published between 2010 and 2019 were part of this review. CONCLUSION: Studies have shown that the use of TMR in post-stroke patients has positive effects on lung volumes and capacities, despite have a small sample size. The search for creating new studies that can assist in the prescription and standardization of the training of these individuals in different environments should be a way to think.

KEYWORDS: Exercise for respiratory muscles. Stroke. Breathing exercises. 


\section{Introdução}

O Acidente Vascular Cerebral (AVC) é o resultado da interrupção aguda do suprimento sanguíneo ao cérebro por meio de entupimento ou rompimento dos vasos $^{1}$ podendo provocar lesões celulares e alterações nas funções neurológicas. Segundo o Ministério da Saúde ${ }^{2}$ em 2017, estatísticas brasileiras indicam que o AVC é a causa mais frequente de óbito na população adulta (cerca de $10 \%$ das mortes) e consiste no diagnóstico de $10 \%$ das internações hospitalares públicas. Em todo o mundo, o AVC está classificado como a segunda maior causa de morte, com cerca de 5,5 milhões de pessoas a cada ano, onde $50 \%$ dos sobreviventes possuem incapacidades crônicas ${ }^{3}$.

O AVC causa comprometimento na produção de estímulo motor, tendo como consequência a perda da função muscular periférica, diminuindo a força dos músculos respiratórios e o fluxo da tosse em $50 \% 4$. As alterações dos componentes após o AVC são relevantes para a ineficiência dos mecanismos orofaríngeos da deglutição, respiração, fonação e reflexos protetores das vias aéreas inferiores culminando em alta incidência de pneumonia por aspiração ${ }^{5}$. Nesse perfil de indivíduos a complacência torácica e pulmonar encontra-se diminuídas o que provoca uma diminuição da capacidade pulmonar total e da capacidade vital ${ }^{\underline{6}}$.

O Treinamento Muscular Respiratório (TMR)* é um conjunto de exercícios capazes de melhorar a funcionalidade da musculatura respiratória. Utilizado por fisioterapeutas na prática clínica, o TMR envolve padrões respiratórios que podem ser combinados com os movimentos dos membros superiores e tronco, bem como manobras na caixa torácica, com ou sem a resistência de um aparelho ${ }^{7}$. Têm como objetivo melhorar o padrão respiratório, aumentar a expansão pulmonar, a capacidade residual funcional e o volume de reserva inspiratório, treinar tanto a força quanto a endurance muscular respiratória, restabelecendo a função muscular quer esteja comprometida ou que tenha forte risco de declinar, melhorar a eficácia na desobstrução das vias aéreas, por meio de uma tosse mais eficiente, além de, através de um condicionamento e adaptação ao exercício, prevenir a fadiga muscular respiratória-10.
Pouco se tem estudado sobre o efeito do treinamento muscular respiratório nas comorbidades respiratórias de pacientes pós-AVC. Sendo assim, o objetivo desse estudo é sistematizar o efeito do treinamento muscular respiratório sobre os volumes e capacidades pulmonares em indivíduos que sofreram acidente vascular cerebral.

\section{Materiais e métodos}

Esta revisão sistemática foi realizada de acordo com os critérios do PRISMA11, por quatro investigadores (PROSPERO - CRD42020193421).

\section{Critérios de Elegibilidade}

Foram incluídos na pesquisa os artigos que atenderam aos seguintes critérios: Ensaios clínicos controlados randomizados, participantes maiores de 18 anos e pós-AVC, além de estudos que utilizem algum método de treinamento muscular respiratório. Foram excluídos os artigos repetidos, os de revisão, trabalhos que não mantiveram relação com o objetivo deste estudo, além de estudos com outras terapêuticas isoladas, que não estivessem associadas ao treinamento muscular respiratório.

\section{Fontes de Informação}

Os artigos foram pesquisados nas bases de dados BVS (Scielo, Lilacs, IBECS, Medline) e PubMed entre o período de janeiro de 2019 a junho de 2020, sem limitação no idioma ou no período em que os estudos foram publicados. Foram utilizados descritores contidos nos Descritores em Ciência da Saúde (DeCS) e Medical Subject Headings (MeSH), nos idiomas de português, inglês e espanhol em combinação com os operadores boleanos AND e OR, além da palavra chave Treinamento Muscular Respiratório/ Respiratory muscle training/ entrenamiento muscular respiratório. Após a seleção nas bases de dados, suas referências foram analisadas e caso houvesse estudos com potencial para fazer parte desse estudo, os mesmos também eram selecionados.

*McConnell A. Breathe strong, perform better. Champaign: Human Kinetics Books; 2011 apud (7). 


\section{Busca}

Os descritores utilizados para busca na BVS foram: ("Acidente Vascular Cerebral" OR "Derrame Cerebral" $O R$ "Ictus Cerebral" OR AVC OR Apoplexia OR "Acidente Cerebrovascular" $O R$ "Apoplexia Cerebrovascular" $O R$ "Icto Cerebral" $O R$ "Acidente Vascular Encefálico" $O R$ AVE $O R$ "Acidente Vascular do Cérebro" $O R$ "Acidente Cerebral Vascular" $O R$ "Acidentes Cerebrais Vasculares" $O R$ "Acidentes Cerebrovasculares" $O R$ "Acidentes Vasculares Cerebrais") AND ("Exercícios Respiratórios" $O R$ "Exercícios para os Músculos Respiratórios" $O R$ "Exercício Respiratório").

Quando buscado pela PubMed os estudos em inglês foram selecionados pelo Mesh Stroke ou seus correlatos (Strokes, Cerebrovascular Accident, Cerebrovascular Accidents, CVA (Cerebrovascular Accident), CVAs (Cerebrovascular Accident), Cerebrovascular Apoplexy, Apoplexy, Cerebrovascular, Vascular Accident, Brain, Brain Vascular Accident, Brain Vascular Accidents, Vascular Accidents, Brain, Cerebrovascular Stroke, Cerebrovascular Strokes, Stroke, Cerebrovascular, Strokes, Cerebrovascular, Apoplexy Cerebral Stroke, Cerebral Strokes, Stroke, Cerebral, Strokes, Cerebral, Stroke, Acute, Acute Stroke, Acute Strokes, Strokes, Acute, Cerebrovascular Accident, Acute, Acute Cerebrovascular Accident, Acute Cerebrovascular Accidents, Cerebrovascular Accidents, Acute), juntamente com Breathing Exercises ou seus correlatos (Exercise, Breathing, Respiratory Muscle Training, Muscle Training, Respiratory, Training, Respiratory Muscle). Para estudos em espanhol os estudos foram selecionados através dos Mesh Accidente Cerebrovascular and Ejercicios Respiratorios.

\section{Seleção dos Estudos}

Os estudos foram rastreados nas bases de dados supracitadas. Inicialmente a seleção dos estudos ocorreu através da verificação dos títulos dos estudos, bem como pela análise dos resumos disponíveis. Caso o estudo fosse selecionado todo o seu conteúdo era lido na íntegra e formou a tabela de resultados dessa revisão.

\section{Processo de Coleta de Dados}

Os estudos selecionados eram analisados conforme a metodologia utilizada e demonstração de resultados. Após a definição da estratégia de busca e padronização de coleta dos autores, o primeiro investigador fez a busca nas bases de dados e selecionou os estudos que fariam parte da revisão. A partir daí os dois autores isoladamente analisaram os estudos e definiram os que continuariam no estudo. Caso houvesse divergência na definição de um estudo, um terceiro investigador fazia a análise independente e decidia por manutenção ou exclusão do mesmo na revisão.

\section{Lista dos Dados}

Após a busca dos artigos os mesmos foram catalogados em planilhas no Microsoft Excel 2010, contendo autor, ano, revista e título. Conforme o estudo era analisado seus dados foram organizados em uma tabela no Microsoft Word 2010, contendo as variáveis: autor e ano da publicação, tamanho da amostra, grupos, desfechos selecionados, intervenção dos grupos e resultados.

\section{Risco de Viés em Cada Estudo}

Os estudos foram avaliados conforme o Manual Cochrane para Desenvolvimento de Revisões Sistemáticas de Intervenção, versão 5.1.0 (Cochrane Handbook) ${ }^{12}$. Esse manual é uma ferramenta que usa domínios para promover uma avaliação crítica sobre diferentes aspectos de riscos de viés. Foi desenvolvido entre 2005 e 2007 por um grupo de editores e autores de revisão sistemática.

É composto por duas partes, contemplados em sete domínios: geração da sequência aleatória, ocultação da alocação, cegamento de participantes e profissionais, cegamento de avaliadores de desfecho, desfechos incompletos, relato de desfecho seletivo e outras fontes de vieses. A primeira parte refere-se à descrição do que foi relatado no estudo que está sendo avaliado. A segunda parte é o julgamento quanto ao risco de viés para cada um dos domínios. Após análise, os domínios podem ser classificados em três categorias: baixo risco de viés, alto risco de viés ou risco de viés incerto ${ }^{13}$. 


\section{Resultados}

Um total de 4834 estudos foi rastreado e analisados após a busca em base de dados e as referências dos estudos analisados. Após análise de elegibilidade através dos critérios de inclusão e exclusão fizeram parte desta revisão 17 estudos (Figura 1).

Figura 1. Seleção dos estudos para análise

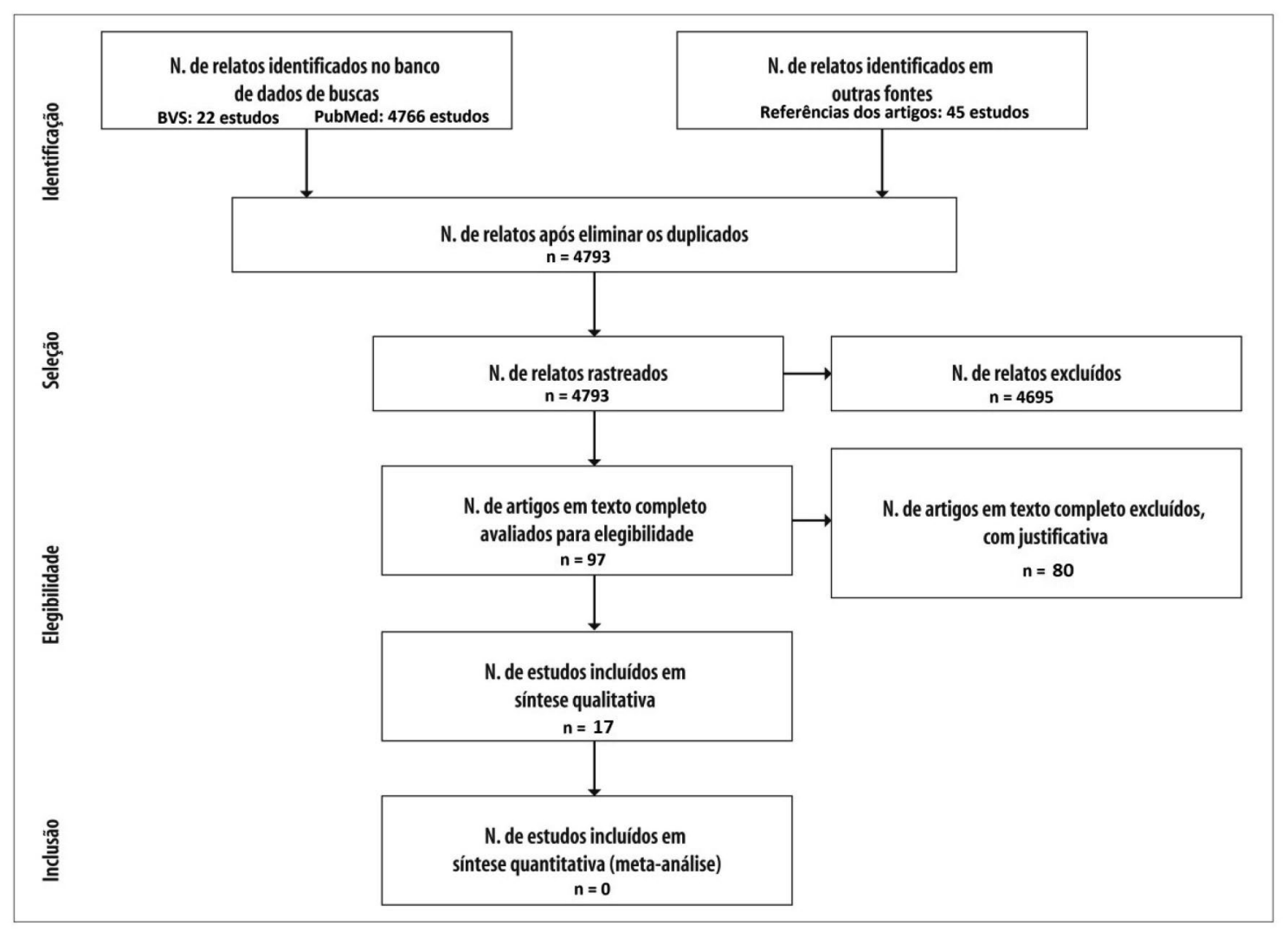

O intervalo de anos das publicações dos estudos variou entre 2010 e 2019 . O tamanho amostral da população variou de 12 até 82 participantes onde na sua grande maioria foram utilizados apenas dois grupos, um grupo experimental e um grupo controle. As exceções ocorreram em três estudos, um incluiu um terceiro grupo sham ${ }^{16}$, e os outros dois incluíram mais um grupo realizando outro método terapêutico ${ }^{14,18}$.

Os principais desfechos de característica respiratória na qual os estudos focaram seus resultados contemplaram principalmente volumes, capacidades e fluxo. Todos os 17 estudos buscaram como objeto de estudo os volumes pulmonares, 15 buscaram capacidades pulmonares, e outros 11 estudos tiveram como desfecho de estudo o fluxo expiratório (Tabela 1). 


\begin{tabular}{|c|c|c|c|c|c|c|}
\hline AUTOR & ANO & AMOSTRA & GRUPOS & $\begin{array}{c}\text { DESFECHOS } \\
\text { SELECIONADOS }\end{array}$ & INTERVENÇÃO & RESULTADOS \\
\hline Kim et $\mathrm{al}^{14}$ & 2015 & 37 & $\begin{array}{c}\mathrm{TI}=13 \\
\mathrm{TMR}=15 \\
\mathrm{CG}=12\end{array}$ & CVF e VEF1. & R-AVC $\times$ TMR $\times$ MA & (+) CVF e VEF1. \\
\hline $\begin{array}{l}\text { Joo, Shin, } \\
\text { Song }{ }^{15}\end{array}$ & 2015 & 38 & $\begin{array}{l}E G=19 \\
C G=19\end{array}$ & $\begin{array}{c}\text { CVF, VEF1, } \\
\text { VEF1/CVF e WM. }\end{array}$ & Game x R-AVC & $\begin{array}{c}\text { (+) CVF, VEF1 e } \\
\text { VVM. }\end{array}$ \\
\hline $\begin{array}{l}\text { Kulnik et } \\
\text { al }^{16}\end{array}$ & 2015 & 82 & $\begin{array}{c}\mathrm{TMI}=27 \\
\mathrm{TME}=26 \\
\text { Sham }=25\end{array}$ & PFE. & TME X TMI x Sham & (+) PFE \\
\hline $\begin{array}{l}\text { Kim, Park, } \\
\text { Yim }^{17}\end{array}$ & 2014 & 20 & $\begin{array}{l}E G=10 \\
C G=10\end{array}$ & $\begin{array}{c}\text { CVF, VEF1, } \\
\text { VEF1/CVF, PFE, }\end{array}$ & TMR $\times$ R-AVC & $\begin{array}{c}(+) \text { CVF, VFE1 } \\
\text { PFE. }\end{array}$ \\
\hline $\begin{array}{l}\text { Lima et } \\
\text { al }^{18}\end{array}$ & 2014 & 40 & $\begin{array}{l}E G=20 \\
C G=20\end{array}$ & CVF, VEF1, VC. & TMR & $(+) \vee C$ \\
\hline $\begin{array}{l}\text { Sutbeyaz } \\
\text { et } \mathrm{al}^{19}\end{array}$ & 2010 & 45 & $\begin{array}{l}\mathrm{TMI}=15 \\
\mathrm{ER}=15 \\
\mathrm{CG}=15\end{array}$ & TFP. & R-AVC x TMR & $\begin{array}{l}\text { (+) VEF1, CVF, } \\
\text { CV, FEF 25-75\%, } \\
\text { VVM, PFE, }\end{array}$ \\
\hline $\begin{array}{l}\text { Jung, } \\
\text { Bang }^{20}\end{array}$ & 2017 & 12 & $\begin{array}{l}E G=6 \\
C G=6\end{array}$ & CVF, VEF1. & TMI $\times$ R-AVC & $(+)$ CVF, VEF1. \\
\hline Jung et $a^{21}$ & 2014 & 18 & $\begin{array}{l}E G=9 \\
C G=9\end{array}$ & $\begin{array}{l}\text { CVF, VEF1, PFE, } \\
\text { FEF25-75\%. }\end{array}$ & $\begin{array}{l}\text { TMI x estimulação } \\
\text { abdominal }\end{array}$ & (+) PFE e VEF1 \\
\hline $\begin{array}{c}\text { Chen et } \\
\mathrm{al}^{22}\end{array}$ & 2016 & 21 & $\begin{array}{l}E G=11 \\
C G=10\end{array}$ & TFP. & R-AVC $\times$ TMI & $(-)$ \\
\hline Jo, $\mathrm{Kim}^{23}$ & 2017 & 25 & $\begin{array}{l}E G=12 \\
C G=13\end{array}$ & CVF e PFE. & R-AVC x TMR & (+) CVF e PFE. \\
\hline $\begin{array}{l}\text { Yoo, } \\
\text { Pyun }^{24}\end{array}$ & 2018 & 40 & $\begin{array}{l}E G=20 \\
C G=20\end{array}$ & CVF, VEF1, PFE. & R-AVC x TMR & (+) CVF, PFE. \\
\hline Jo, $\mathrm{Kim}^{25}$ & 2016 & 42 & $\begin{array}{l}E G=21 \\
C G=21\end{array}$ & CVF e PFE. & R-AVC x TMR & (+) CVF, PFE. \\
\hline $\begin{array}{l}\text { Lee, Park, } \\
\text { Lee }^{26}\end{array}$ & 2019 & 25 & $\begin{array}{l}E G=13 \\
C G=12\end{array}$ & $\begin{array}{l}\text { PFE, VEF1, PFI, } \\
\text { CVF. }\end{array}$ & R-AVC $x$ TMR & $\begin{array}{c}\text { (+) PEM, PFE, } \\
\text { PFI. }\end{array}$ \\
\hline Jung, Kim ${ }^{27}$ & 2013 & 29 & $\begin{array}{l}E G=15 \\
C G=14\end{array}$ & $\begin{array}{l}\text { VEF1, PFE e } \\
\text { VEF1/CVF. }\end{array}$ & R-AVC $\times$ TMI & $(-)$ \\
\hline Kim, Lee ${ }^{28}$ & 2018 & 24 & $\begin{array}{l}E G=12 \\
C G=12\end{array}$ & CVF e VEF1. & R-AVC $\times$ TMR & (+) CVF e VEF1. \\
\hline $\begin{array}{l}\text { Kim, Lee, } \\
\text { Yun }^{29}\end{array}$ & 2012 & 18 & $\begin{array}{l}E G=10 \\
C G=8\end{array}$ & $\begin{array}{c}\text { CVF, VEF1, } \\
\text { VEF1/CVF, PFE. }\end{array}$ & R-AVC $x$ TMR & $\begin{array}{c}(+) \text { VEF1, CVF, } \\
\text { PFE. }\end{array}$ \\
\hline $\begin{array}{l}\text { Jo, Kim, } \\
\text { Jung }\end{array}$ & 2014 & 34 & $\begin{array}{l}E G=17 \\
C G=17\end{array}$ & CVF e PFE. & R-AVC $\times$ TMR & (+) CVF e PFE. \\
\hline \multicolumn{7}{|c|}{$\begin{array}{l}\text { Legenda: EG = Grupo experimental; CG: Grupo controle; TI = Treinamento integrado; CVF = Capacidade vital } \\
\text { forçada; VEF1 = Volume expiratório final } 1 \text { segundo; PFE = Pico de fluxo expiratório; PFI = Pico de fluxo } \\
\text { inspiratório; VVM = Volume ventilatório máximo; CV = Capacidade vital; FEF = Fluxo expiratório forçado; VC = } \\
\text { Volume corrente; TFP = Teste de função pulmonar; TMI = Treinamento muscular inspiratório; TME = Treinamento } \\
\text { muscular expiratório; TMR = Treinamento muscular respiratório; R-AVC: Reabilitação para Acidente Vascular } \\
\text { Cerebral; MA = Manobra abdominal. }\end{array}$} \\
\hline
\end{tabular}


Ao todo, 16 estudos utilizaram dispositivos para o treinamento muscular respiratório, que podia ser do tipo inspiratório, expiratório ou por espirometria de incentivo. Dois estudos utilizaram de exercícios respiratórios abdominal em algum dos grupos e um utilizou exercícios baseados em um game como método de treinamento. A fisioterapia convencional, ou cinesioterapia, no AVC foi o método de tratamento mais utilizado no grupo controle, não sendo utilizada em apenas 3 dos estudos.

Se tratando da padronização do treinamento utilizado, 10 estudos graduaram a sessão por minutos executados entre 15 e 30 minutos, enquanto outros 7 eram por repetições realizadas, variando entre 3 e 30 repetições. Sobre a divisão em séries da sessão, 8 estudos fizeram a abordagem em apenas uma série, 2 estudos dividiram em 2 séries, 3 estudos dividiram em 3 séries, 3 dividiram em 5 séries e apenas 1 dividiu em 6 séries. Enquanto três estudos não limitaram a quantidade de sessões durante a semana, os outros 14 estudos variaram entre 3 e 7 vezes na semana. No total de semanas de treinamento a variação dos estudos foi de três a dez semanas, porém, apenas 1 estudo fez a abordagem em um único dia (Tabela 2).

Tabela 2. Utilização do TMR pelos estudos analisados

\begin{tabular}{|c|c|c|c|c|c|}
\hline AUTOR & REPETIÇÕES & SÉRIES & TEMPO & CARGA (f, p) & $\begin{array}{l}\text { TEMPO TOTAL DE } \\
\text { INTERVENÇÃO }\end{array}$ \\
\hline Kim et al ${ }^{14}$ & 10 & 5 & $5 x /$ sem & - & 6 sem \\
\hline Joo, Shin, Song ${ }^{15}$ & $25 \mathrm{~min}$ & - & $3 x /$ sem & $f$ & 5 sem \\
\hline Kulnik et al ${ }^{16}$ & 10 & 5 & $x$ & $\begin{array}{l}\text { p 50\% pimáx ou } \\
\text { pemáx }\end{array}$ & 4 sem \\
\hline Kim, Park, Yim ${ }^{17}$ & $20 \mathrm{~min}$ & - & $3 x /$ sem & $p$ & 4 sem \\
\hline Lima et $\mathrm{al}^{18}$ & 10 & 3 & $x$ & 80\% pimáx & $1 \mathrm{dia}$ \\
\hline Sutbeyaz et al ${ }^{19}$ & $15 \mathrm{~min}$ & 2 & $6 x /$ sem & p 40\% pimáx & 6 sem \\
\hline Jung, Bang ${ }^{20}$ & $5 \mathrm{~min}$ & 6 & $5 x /$ sem & f 30\% pimáx & 4sem \\
\hline Jung et $\mathbf{a l}^{21}$ & $20 \mathrm{~min}$ & - & $3 x /$ sem & f 30\%pimáx & 4 sem \\
\hline Chen et $\mathrm{al}^{22}$ & 30 & - & $5 x /$ sem & p 30\% pimáx & 10 sem \\
\hline Jo, Kim $^{23}$ & 20-30min & - & $3 x /$ sem & $\begin{array}{l}\text { p pimáx ou } \\
\text { pemáx }\end{array}$ & 8 sem \\
\hline Yoo, Pyun ${ }^{24}$ & $30 \mathrm{~min}$ & 2 & $7 x /$ sem & - & 3sem \\
\hline Jo, $\operatorname{Kim}^{25}$ & 3 & 3 & $x$ & $\begin{array}{l}\text { p pimáx ou } \\
\text { pemáx }\end{array}$ & 8 sem \\
\hline Lee, Park, Lee ${ }^{26}$ & $10-15$ & 5 & $3 x /$ sem & p 30\% pimáx & $6 s e m$ \\
\hline Jung, Kim²7 & $20 \mathrm{~min}$ & - & $3 x /$ sem & - & 6 sem \\
\hline Kim, Lee ${ }^{28}$ & $20 \mathrm{~min}$ & - & $5 x / \min$ & p & 4sem \\
\hline Kim, Lee, Yun ${ }^{29}$ & $30 \mathrm{~min}$ & - & $3 x /$ sem & $p$ & 6 sem \\
\hline Jo, Kim, Jung ${ }^{30}$ & 3 & 3 & $3 x /$ sem & P pimáx ou pemáx & 4 sem \\
\hline
\end{tabular}

Dos estudos analisados quanto ao risco de viés, apenas dois não apresentaram risco alto ou incerto. Houve 11 estudos que deixaram em dúvida a análise de pontos da escala avaliadora, o que fez com que recebessem como avaliação o risco de viés incerto. Sobre as características importantes a um ensaio clinico que minimizem as interferências quanto aos resultados encontrados, 13 estudos não as cumpriram em sua totalidade, recebendo um escore de alto risco de viés (Gráfico 1). 


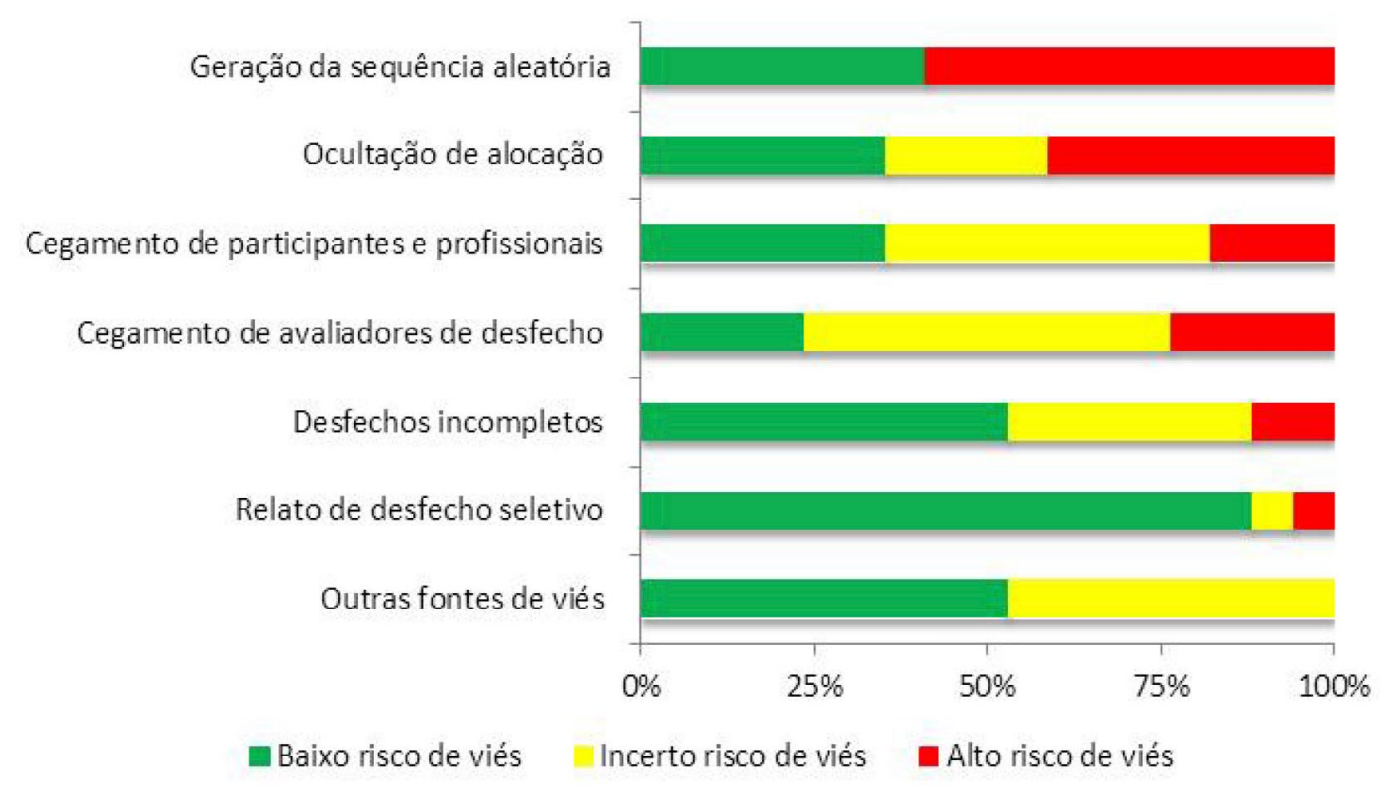

\section{Discussão}

Já é sabido que nesse perfil de indivíduos acontece um decréscimo no valor da Capacidade Vital Forçada (CVF) comparado ao valor de referência ${ }^{6}$, que podem gerar distúrbios ventilatórios graves em longo prazo ${ }^{31}$. Sendo assim, 10 estudos concluíram que após a utilização do TMR em indivíduos pós-AVC há uma melhora significativa na CVF. Esse volume de ar exalado é obtido quando se imprime máximo esforço a partir de uma inspiração má$x_{i m a}{ }^{31}$. O TMR pode ser utilizado nesses pacientes visando melhora nos volumes, que geralmente encontram-se reduzidos por conta do desequilíbrio muscular que proporciona a entrada desse ar nos pulmões ${ }^{32}$.

O Volume Expiratório Forçado no Primeiro Segundo (VEF1) representa a quantidade de ar que sai no primeiro segundo da CVF. Seu valor destaca se há obstrução da saída de ar, fazendo com que o mesmo permaneça por mais tempo retido no pulmão, tendo impacto importante na oxigenação e consequentemente nas atividades aeróbicas ${ }^{33}$. Sete (07) estudos dessa revisão concluíram que o TMR como técnica pra tratamento de distúrbios ventilatórios se mostrou eficaz na melhora do VEF1. Com isso é possível ter base para utilização do TMR quando os pacientes pósAVC apresentem distúrbios obstrutivos, beneficiando-se de mais uma opção pra melhora das atividades diárias.

Mesmo com um número considerável de estudos mostrando a melhora do VEF1 e CVF, 5 estudos não acharam melhoras em algum desses marcadores: 4 não acharam melhora significativa em CVF, e 3 no VEF1. Em um estudo $\frac{18}{}$ foi realizado uma técnica de espirometria de incentivo com 3 séries de 10 repetições em apenas um dia, onde o objetivo era verificar o efeito agudo da técnica em pacientes pós-AVC. Esse tempo pode ter influenciado no resultado final, além de que seu grupo controle foi formado por indivíduos saudáveis, que também obtém meIhora na capacidade ventilatória após o treinamento ${ }^{34}$. Além disso, o corpo humano tende a fazer compensações em outras variáveis pra manter os valores mais próximos da normalidade, nesse caso modificando a frequência respiratória e o volume minuto para compensar nas trocas gasosas ${ }^{6}$.

Se tratando de Volume Ventilatório Máximo (VVM) foi possível verificar a melhora nesse marcador através da análise de 2 estudos. A VVM é um volume obtido através de técnica repetitiva e dinâmica de contrações musculares respiratórias, ocorrendo expansão e retração torácica de modo rápido e brusco. Como é uma técnica que precisa de força muscular integra, seus valores baixos refletem doenças restritivas clinicamente significantes ${ }^{31}$. Os indivíduos pós-AVC, que apresentam desequilíbrio muscular, uma maior rigidez da caixa torácica e baixa tolerância a atividades aeróbicas prolongadas ${ }^{\circ}$, se beneficiam do TMR por promover maior tolerância e qualidade em atividades de baixa e moderada intensidade. 
Pensando em Volume Corrente (VC), apenas um estudo utilizou esse marcador como objeto de análise e pode constatar a melhora dele ao final da aplicação do TMR. Sendo o VC a quantidade de ar que entra e sai dos pulmões numa ventilação basal, ele é importante pra manutenção das trocas em períodos de repouso ${ }^{31}$. Devido às alterações de caixa torácica e musculares no pós-AVC já citadas, trabalhar a musculatura pra que ela precise gerar menos esforço para manter as trocas basais se tornam importantes pra essa população, gerando menor gasto energético em repouso.

Dos estudos verificados, 11 trataram de analisar o Pico de Fluxo Expiratório (PFE), tendo como resposta a confirmação do TMR como técnica pra melhora desse marcador. O PFE é obtido de uma rápida exalação de ar após uma inspiração máxima ${ }^{31}$. Fisiologicamente realizamos isso durante o reflexo de tosse, por isso ele é tão importante pra determinar uma melhor capacidade de tosse ${ }^{35}$. A função do reflexo de tosse é remover a secreção e/ou corpo estranho das vias aéreas, gerando um fluxo expiratório importante na defesa das vias 5 . Ao treinar a musculatura respiratória desses indivíduos reduz-se o risco de comorbidades e mortalidade, já que a pneumonia por aspiração pode ser um fator de óbito pra essa população.

\section{Conclusão}

Esse estudo concluiu que existem diversos efeitos positivos nos volumes e capacidades respiratórias de indivíduos pós-AVC submetidos à utilização de TMR. Há diversos estudos comprovando os efeitos desse treinamento na CVF, VEF1, VVM e PFE. Apesar de muitos estudos terem um tamanho amostral pequeno a confirmação da melhora promovida pelo TMR através desses estudos nos ajuda a ter entendimento da utilização desse método na nossa pratica clínica, visando evitar os efeitos deletérios causados pelo AVC na função pulmonar.

O uso de TMR em pacientes pós-AVC parece ter chamado atenção de estudiosos mais recentemente na literatura, porém com o passar do tempo mais estudos estão sendo feitos nessa população. Sugere-se a produção de novos estudos que possuam um maior número de participantes e com protocolos distintos que possam auxiliar na prescrição e padronização dos treinamentos desses indivíduos em diversos ambientes.

\section{Contribuições dos autores}

Nascimento JS participou da concepção, delineamento, busca e análise estatística dos dados da pesquisa, interpretação dos resultados e redação do artigo científico. Neto FF participou da concepção, delineamento e revisão da redação final. Ribeiro NMS participou da concepção, delineamento e análise final dos dados. Barauna LH participou da concepção, delineamento e redação. Jesus ACC participou da participou da concepção, delineamento e interpretação de resultados.

\section{Conflitos de interesses}

Nenhum conflito financeiro, legal ou político envolvendo terceiros (governo, empresas e fundações privadas, etc.) foi declarado para nenhum aspecto do trabalho submetido (incluindo, mas não se limitando a subvenções e financiamentos, participação em conselho consultivo, desenho de estudo, preparação de manuscrito, análise estatística, etc.).

\section{Referências}

1. Biblioteca Virtual em Saúde. AVC - Acidente Vascular Cerebral. [Internet]. 2006. [acesso em 2020 jul 27]. Disponível em: https:// bvsms.saude.gov.br/bvs/dicas/105avc.html

2. Ministério da Saúde. Acidente vascular cerebral - AVC. [Internet]. [acesso em 2018 set 07]. Disponível em: http://bvsms. saude.gov.br/dicas-em-saude/2188-avc-acidente-vascular-cerebral

3. Donkor ES. Stroke in the 21st Century: A Snapshot of the Burden, Epidemiology, and Quality of Life. Stroke Res Treat. 2018; 3238165. doi: $10.1155 / 2018 / 3238165$

4. Pompeu SMAA, Pompeu JE, Rosa M, Silva MR. Correlação entre função motora, equilíbrio e força respiratória pós Acidente Vascular Cerebral. Rev Neurocienc. 2011;19(4): 614-20. doi: 10.34024/rnc.2011.v19.8324

5. Gasparim AZ, Jurkiewicz AL, Marques JM, Santos RS, Marcelino PCO, Herrero Junior F. Deglutição e tosse nos diferentes graus da doença de parkinson. Arq Int Otorrinolaringol. 2011;15(2):181-88. doi: 10.1590/S1809-48722011000200010

6. Cury JL, Pinheiro AR, Brunetto AF. Modificações da dinâmica respiratória em indivíduos com hemiparesia pós-acidente vascular encefálico. Assobrafir Ciência. 2009;1(1):55-68. doi: 10.47066/2177-9333/ac.4185

7. Siqueira VS. Treinamento muscular respiratório: Uma abordagem teórico-prática no contexto da promoção da saúde [dissertação]. Paraná: Universidade Norte do Paraná; 2018.

8. Barros GF, Santos CS, Granado FB, Costa PT, Límaco RP, Gardenghi G. Treinamento muscular respiratório na revascularização do miocárdio. Rev Bras Cir Cardiovasc. 2010; 25(4):483-90. doi: 10.1590/S0102-76382010000400011 
9. Pascotini FS, Denardi C, Nunes GO, Trevisan ME, Antunes VP. Treinamento muscular respiratório em pacientes em desmame da ventilação mecânica. ABCS Health Sci. 2014; 39(1):12-16. doi: 10.7322/abcshs.v39i1.253

10. Grams ST, Ono LM, Noronha MA, Schivinsk CIS, Paulin E. Breathing exercises in upper abdominal surgery: a systematic review and meta-analysis. Rev Bras Fisioter. 2012;16(5): 345-53. doi: 10.1590/S1413-35552012005000052

11. Galvão TF, Pansani TSA, Harrad D. Principais itens para relatar Revisões sistemáticas e Meta-análises: A recomendação PRISMA. Epidemiol Serv Saúde. 2015;24(2):335-42. doi: 10.5123/S167949742015000200017

12. Ministério da Saúde. Diretrizes metodológicas: elaboração de revisão sistemática e metanálise de ensaios clínicos randomizados. [Internet]. 2012. [acesso em 2019 mai 06]. Disponível em http://bvsms.saude.gov.br/bvs/publicacoes/ diretrizes_metodologicas_elaboracao_sistematica.pdf

13. Carvalho APV, Silva V, Grande AJ. Avaliação do risco de viés de ensaios clínicos randomizados pela ferramenta da colaboração Cochrane. Diagn Tratamento. 2013;18(1):38-44.

14. Kim CY, Lee JS, Kim HD, Kim IS. Effects of the combination of respiratory muscle training and abdominal drawing-in maneuver on respiratory muscle activity in patients with post-stroke hemiplegia: a pilot randomized controlled trial. Top Stroke Rehabil. 2015; 22(4):262-70. doi: $\underline{10.1179 / 1074935714 Z .0000000020}$

15. Joo S, Shin D, Song C. The effects of game-based breathing exercise on pulmonary function in stroke patients: a preliminary study. Med Sci Monit. 2015;21:1806-11. doi: 10.12659/ MSM.893420

16. Kulnik ST, Birring SS, Moxham J, Rafferty GF, Kalra L. Does respiratory muscle training improve cough flow in acute stroke? Pilot randomized controlled trial. Stroke. 2014; 46(2):447-53. doi: 10.1161/STROKEAHA.114.007110

17. Kim JH, Park JH, Yim J. Effects of respiratory muscle and endurance training using an individualized training device on pulmonary function and exercise capacity in stroke patients. Med Sci Monit. 2014;20:2543-49. doi: 10.12659/MSM.891112

18. Lima IN, Fregonezi GA, Melo R, Cabral EE, Aliverti A, Campos TF et al. Acute effects of volume-oriented incentive spirometry on chest wall volumes in patients after a stroke. Resp Care. 2014;59(7):1101-7. doi: 10.4187/respcare.02651

19. Sutbeyaz ST, Koseoglu F, Inan L, Coskun O. Respiratory muscle training improves cardiopulmonary function and exercise tolerance in subjects with subacute stroke: a randomized controlled trial. Clin Rehab. 2010;24(3):240-50. doi: 10.1177/0269215509358932
20. Jung KM, Bang DH. Effect of inspiratory muscle training on respiratory capacity and walking ability with subacute stroke patients: a randomized controlled pilot trial. J Phys Ther Sci. 2017;29(2):336-39. doi: 10.1589/jpts.29.336

21. Jung JH, Shim JM, Kwon HY, Kim HR, Kim BI. Effects of abdominal stimulation during inspiratory muscle training on respiratory function of chronic stroke patients. J Phys Ther Sci. 2014;26(1):73-76. doi: 10.1589/jpts.26.73

22. Chen PC, Liaw MY, Wang LY, Tsai YC, Hsin YJ, Chen YC et al. Inspiratory muscle training in stroke patients with congestive heart failure: A CONSORT-compliant prospective randomized single-blind controlled trial. Medic. 2016;95(37):e4856. doi: 10.1097/MD.0000000000004856

23. Jo MR, Kim NS. Combined respiratory muscle training facilitates expiratory muscle activity in stroke patients. J Phys Ther Sci. 2017;29(11):1970-973. doi: 10.1589/jpts.29.1970

24. Yoo HJ, Pyun SB. Efficacy of bedside respiratory muscle training in patients with stroke: A randomized controlled trial. Amer J Phys Med Rehabil. 2010;97(10):691-97. doi: 10.1097/ PHM.0000000000000933

25. Jo MR, Kim NS. The correlation of respiratory muscle strength and cough capacity in stroke patients. J Phys Ther Sci. 2016. 28(10):2803-805. doi: 10.1589/jpts.28.2803

26. Lee K, Park D, Lee G. Progressive respiratory muscle training for improving trunk stability in chronic stroke survivors: A pilot randomized controlled trial. J Stroke Cerebrovasc Dis. 2019;28(5):1200-1211. doi: 10.1016/j. jstrokecerebrovasdis.2019.01.008

27. Jung JH, Kim NS. Effects of inspiratory muscle training on diaphragm thickness, pulmonary function, and chest expansion in chronic stroke patients. J The Kor Soc of Phys Medic. 2013;8(1):5969. doi: $10.13066 / \mathrm{kspm} .2013 .8 .1 .059$

28. Lee DK, Kim SH. The effect of respiratory exercise on trunk control, pulmonary function, and trunk muscle activity in chronic stroke patients. J Phys Ther Sci. 2018;30:700-703. doi: 10.1589/ jpts.30.700

29. Kim MH, Lee WH, Yun MJ. The effects on respiratory strength training on respiratory function and trunk control in patient with stroke. J Korean Soc Phys Ther. 2012;24(5):340-47.

30. Jo MR, Kim NS, Jung JH. The effects of respiratory muscle training on respiratory function, respiratory muscle strength, and cough capacity in stroke patients. J Korean Soc Phys Med. 2014;9(4):399-406. doi: 10.13066/kspm.2014.9.4.399

31. Pereira CAC. Espirometria. J Pneumol. 2002; 28(Suppl 3): S1S238. 
32. Mafalda L, Santos PH, Carrilho LO. Perfil respiratório de pacientes acometidos por acidente vascular encefálico. Saúde Integrada. 2015; 07:30-48.

33. Pereira CAC. Bases e aplicações clínicas dos testes de função pulmonar. Rev Bras Med Trab. 2004; 2(4): 317-30.

34. Reis IMM, Pessoa-Santos BV, Basso-Vanelli RP, Di Lorenzo VAP, Jamami M. Efeitos do treinamento com espirômetros de incentivo a fluxo e a volume em indivíduos saudáveis. $\mathrm{R}$ Bras Ci e Mov.

2015;23(2):104-12. doi: 10.18511/rbcm.v23i2.5217
35. Meireles ALF, Meireles LCF, Queiroz JCES, Tassitano RM, Soares FO, Oliveira AS. Eficácia da eletroestimulação muscular expiratória na tosse de pacientes após acidente vascular encefálico. Fisioter Pesq. 2012;19(4):314-19. doi: 10.1590/S1809-29502012000400004 